\title{
HYPERBOLIC SUBMANIFOLDS OF COMPLEX PROJECTIVE SPACE
}

\author{
PETER KIERNAN
}

In [1] Professor Kobayashi constructed an invariant pseudodistance $d_{M}$ on each complex manifold $M$. If the pseudo-distance $d_{M}$ is a true distance, the complex manifold is said to be hyperbolic. It is known (see [1]) that if $M$ admits a hermitian metric of strongly negative curvature then $M$ is hyperbolic. In this paper, examples of hyperbolic manifolds are obtained by a more elementary method. In the process, it shall be shown that any covering manifold of the complement of any $2 n$ hyperplanes in general position in $P_{n}(C)$ is not biholomorphically equivalent to a bounded domain of $C^{n}$. This gives a negative answer to a question posed by Professor Chern.

Before proceeding, it will be useful to state some of Kobayashi's basic results concerning the pseudo-distance $d_{M}$. For further details and proofs, see [1].

THEOREM 1. If $f: M \rightarrow N$ is holomorphic, then $f$ is distance decreasing with respect to $d_{M}$ and $d_{N}$. Thus if $N$ is hyperbolic and $d_{M} \equiv 0$, then $f$ is constant. If $N$ is hyperbolic and there exists a 1-1 holomorphic mapping $f: M \rightarrow N$, then $M$ is hyperbolic.

TheOREM 2. Let $\tilde{M}$ be a covering manifold of $M$. Then $\tilde{M}$ is hyperbolic if and only if $M$ is hyperbolic.

Theorem 3. If $M$ and $N$ are hyperbolic, then $M \times N$ is hyperbolic.

Theorem 4. $D^{n}$, the unit disc in $C^{n}$, is hyperbolic. $C-\{0,1\}$ is hyperbolic. $C$ and $C-\{0\}$ are not hyperbolic and, in fact, $d_{C} \equiv 0 \equiv d_{C-\{0\}}$.

The following notation is necessary in order to give the examples. Let

$K_{n}=\left\{\sigma=a_{1} \cup a_{2} \cup a_{3}\right.$ where the $a_{i}$ are distinct hyperplanes in $P_{n}(C)$ which intersect in an $n-2$ dimensional subspace of $\left.P_{n}(C)\right\}$.

$L_{n}=\left\{A=\sigma_{1} \cup \ldots \cup \sigma_{n} \mid \sigma_{\alpha}=a_{\alpha 1} \cup a_{\alpha 2} \cup a_{\alpha 3} \in K_{n}\right.$ and the following conditions are satisfied:

(i) For each $1<\alpha \leqq n$ there exists $\beta<\alpha$ and there exists $(i, k)$ such that $a_{\alpha i}=a_{\beta k}$. Furthermore the $i$ is unique.

(ii) If $b_{\alpha}=a_{\alpha 1} \cap a_{\alpha 2} \cap a_{\alpha 3}$, then for any set of $n$ hyperplanes $\left\{y_{1}, \cdots, y_{n}\right\}$ with $b_{\alpha} \subset y_{\alpha} \nsubseteq A$, we have $y_{1} \cap \cdots \cap y_{n}=$ point $\}$.

Received by the editors January 9, 1969. 
REMARKs. A member of $L_{n}$ is a union of $n$ elements $\sigma_{1}, \cdots, \sigma_{n}$ of $K_{n}$ which satisfy two conditions. The first condition says that exactly one of the hyperplanes of $\sigma_{\alpha}$ is in a previous $\sigma_{\beta}$. Thus an element of $L_{n}$ is the union of $2 n+1$ hyperplanes. The second condition forces the $2 n+1$ hyperplanes to have a nice relative position. For $n=1$ or 2 , the first condition implies the second.

Theorem 5. Let $A \in L_{n}$. Then $M=P_{n}(C)-A$ is hyperbolic.

Proof. Let $p \in M$ and $q \in M$ be such that $d_{M}(p, q)=0$. Let $A=\sigma_{1} \cup \ldots \cup \sigma_{n}, \quad \sigma_{\alpha}=a_{\alpha 1} \cup a_{\alpha 2} \cup a_{\alpha 3}$ and $b_{\alpha}=a_{\alpha 1} \cap a_{\alpha 2} \cap a_{\alpha 3}$. Let $N_{\alpha}=P_{n}(C)-\sigma_{\alpha}$. We can choose coordinates in $N_{\alpha}$ such that

$$
N_{\alpha}=C^{n}-\left\{\left(z_{1}, \cdots, z_{n}\right) \mid z_{n}=0 \text { or } z_{n}=1\right\} .
$$

Define $\phi_{\alpha}: N_{\alpha} \rightarrow C-\{0,1\}$ by $\phi_{\alpha}\left(z_{1}, \cdots, z_{n}\right)=z_{n}$. Since $C-\{0,1\}$ is hyperbolic, this says that $\phi_{\alpha}(p)=\phi_{\alpha}(q)$. Thus there exists a hyperplane $y_{\alpha}$ such that $\{p, q\} \subset y_{\alpha}$ and $b_{\alpha} \subset y_{\alpha} \leftarrow A$. Doing this for all $\alpha$, we have $\{p, q\} \subset y_{1} \cap \cdots \cap y_{n}$. By property (ii) in the definition of $L_{n}$, we have $p=q$ and therefore $M$ is hyperbolic. This completes the proof.

The previous theorem shows that if $A$ is the union of $2 n+1$ hyperplanes in $P_{n}(C)$ which have the proper relative position, then $P_{n}(C)-A$ is hyperbolic. If $A=\sigma_{1} \cup \cdots \cup \sigma_{n} \in L_{n}$ has the property that one hyperplane, say $a_{11}$, is common to all $\sigma_{\alpha}$, then $P_{n}(C)-A$ is equivalent to $C-\{0,1\} \times \cdots \times C-\{0,1\}$. In this case, $A$ is given in homogeneous coordinates by the equation

$$
z_{0} z_{1} \cdots z_{n}\left(z_{0}-z_{n}\right)\left(z_{1}-z_{n}\right) \cdots\left(z_{n-1}-z_{n}\right)=0 .
$$

For $n=1$ or $n=2$, this is the only example we obtain. However, for $n \geqq 3$ we obtain more. For example, each of the following equations define an element of $L_{4}$.

$$
\begin{aligned}
& z_{0} \cdots z_{4}\left(z_{0}-z_{4}\right)\left(z_{1}-z_{4}\right)\left(z_{2}-z_{4}\right)\left(z_{3}-z_{4}\right)=0 \\
& z_{0} \cdots z_{4}\left(z_{0}-z_{4}\right)\left(z_{1}-z_{4}\right)\left(z_{2}-z_{4}\right)\left(z_{2}-z_{3}\right)=0 \\
& z_{0} \cdots z_{4}\left(z_{0}-z_{4}\right)\left(z_{1}-z_{4}\right)\left(z_{1}-z_{2}\right)\left(z_{2}-z_{3}\right)=0 .
\end{aligned}
$$

We now consider the complement of $2 n$ hyperplanes in $P_{n}(C)$. Let $V=a_{1} \cup \ldots \cup a_{2 n}$ be the union of $2 n$ hyperplanes in $P_{n}(C)$. We say that $V$ satisfies property $\mathrm{P}$ if (after reordering the $a_{i}$ if necessary):

(P) There exists points $p$ and $q$ and some $0 \leqq k \leqq 2 n$ such that $p \in a_{1} \cap \cdots \cap a_{k}$ and $q \in a_{k+1} \cap \cdots \cap a_{2 n}$, and such that the line determined by $p$ and $q$ is not contained in any of the hyperplanes $a_{j}$. 
Theorem 6. If $V$ is the union of $2 n$ hyperplanes in $P_{n}(C)$ and $V$ satisfies property $\mathrm{P}$, then $P_{n}(C)-V$ is not hyperbolic.

Proof. Property $\mathrm{P}$ implies that there exists a nonconstant holomorphic map of $C-\{0\}$ into $P_{n}(C)-V$. Thus by Theorems 1 and 4, $P_{n}(C)-V$ is not hyperbolic.

CoROllary 1. If $V$ is the union of $2 n$ hyperplanes in general position in $P_{n}(C)$, then $P_{n}(C)-V$ is not hyperbolic.

Proof. Let $V=a_{1} \cup \cdots \cup a_{2 n}, p \in a_{1} \cap \cdots \cap a_{n}$ and $q \in a_{n+1}$ $\cap \cdots \cap a_{2 n}$. If the line determined by $p$ and $q$ is contained in $a_{k}$, then $a_{1} \cap \cdots \cap a_{n} \cap a_{k} \neq \varnothing$ and $a_{k} \cap a_{n+1} \cap \cdots \cap a_{2 n} \neq \varnothing$. This is impossible since the $a_{i}$ are in general position. Thus $V$ satisfies property P.

COROLlaRY 2. If $n \leqq 5$ and $V$ is the union of any $2 n$ hyperplanes in $P_{n}(C)$, then $P_{n}(C)-V$ is not hyperbolic.

Proof. This is proved by considering the different possible ways in which the hyperplanes could intersect. For example, if $n=2$ and $V=a_{1} \cup \ldots \cup a_{4}$, there are three cases (up to relabelling the $a_{i}$ ):

(1) $a_{1} \cap \cdots \cap a_{4} \neq \varnothing$,

(2) $a_{1} \cap \cdots \cap a_{4}=\varnothing$ and $a_{1} \cap a_{2} \cap a_{3} \neq \varnothing$,

(3) the $a_{i}$ are in general position.

In each case it is easy to show that $V$ satisfies property P. Similar arguments work for $n=3,4$ or 5 .

REMARK. I feel that if $V$ is the union of any $2 n$ hyperplanes in $P_{n}(C)$, then $V$ satisfies property $\mathrm{P}$. This would imply that a minimum of $2 n+1$ hyperplanes must be removed from $P_{n}(C)$ in order to obtain a hyperbolic space. However, the arguments used to prove this for $n \leqq 5$ do not seem to generalize.

COROLlARY 3. Let $V$ be as in Theorem 6 and let $\tilde{M}$ be a covering manifold of $P_{n}(C)-V$. Then any bounded holomorphic map $f: \tilde{M} \rightarrow C$ is not 1-1. In particular, $\tilde{M}$ is not biholomorphically equivalent to a bounded domain of $C^{n}$.

Proof. Any bounded domain is hyperbolic. Theorems 2 and 6 imply that $\tilde{M}$ is not hyperbolic. Therefore Theorem 1 implies that $f$ is not 1-1. This completes the proof.

Let $V$ be a complete quadrilateral in $P_{2}(C)$ with diagonal $T$. Then Corollary 2 says that $M=P_{2}(C)-V$ is not hyperbolic and Corollary 3 says that any covering $\tilde{M}$ of $M$ is not biholomorphically equivalent 
to a bounded domain in $C$. However, $V \cup T \in L_{2}$ and therefore $P_{2}(C)-V \cup T$ is equivalent to $C-\{0,1\} \times C-\{0,1\}$, which is covered by $D^{\prime} \times D^{\prime}$. These last results have been obtained independently by Wilhelm Stoll [2].

We finish with an example. Let $n \geqq 2$ and let $A^{d} C P_{n}(C)$ be the variety defined by the homogeneous equation $z_{0}^{d}+\cdots+z_{n}^{d}=0$ where $d$ is a positive integer. Then $M^{d}=P_{n}(C)-A^{d}$ is not hyperbolic for any $d$. To see this, let $U_{n}$ be the coordinate neighborhood obtained by setting $z_{n}=1$. Then the $\operatorname{map} f: C \rightarrow U_{n}$ defined by $f(z)=\left(z,(-1)^{1 / d} z\right.$, $0, \cdots, 0)$ is nonconstant. Therefore $M^{d}$ is not hyperbolic.

\section{REFERENCES}

1. Shoshichi Kobayashi, Invariant distances on complex manifolds and holomorphic mappings, J. Math. Soc. Japan 19 (1967), 460-480.

2. Wilhelm Stoll, About the universal covering of the complement of a complete quadrilateral, Proc. Amer. Math. Soc. 22 (1969), 326-327.

University of California, Berkeley 